\title{
GOBIERNO ABIERTO, EL VALOR SOCIAL DE LA INFORMACIÓN PÚBLICA
}

\section{Álvaro PORRAS VIVAS}

Actualmente, son escasas las publicaciones académicas que desarrollan de manera clara y exhaustiva lo que implica la conceptualización e implementación del denominado "Gobierno abierto" en México. No obstante, la población se ve constantemente bombardeada a través de los medios de comunicación respecto de notas periodísticas y noticias que hacen referencia a supuestas acciones que sirven para coadyuvar con el desarrollo de un gobierno abierto en México, sin siquiera entender a qué se refieren con ello o cuáles son los alcances, metas y medios para la implementación y desarrollo de este fenómeno dentro del Estado mexicano.

Lo anterior es relevante, toda vez que desde la adhesión del Estado mexicano a la Alianza para el Gobierno Abierto (AGA), ${ }^{* *}$ en septiembre de 2011, nuestro país asumió diversos compromisos que buscan avanzar en materia de transparencia y acceso a la información, participación ciudadana, rendición de cuentas y el uso de las tecnologías de la información. Dichos aspectos, de manera específica, enmarcan el importante papel que juega la ciudadanía dentro del proceso de toma de decisiones de las autoridades mediante el uso de las herramientas tecnológicas.

En ese sentido, no sería posible la participación ciudadana sin la difusión de aquellos aspectos relevantes que coadyuven a que la población mexicana se encuentre informada respecto de las implicaciones y herramientas que les brinda el "Gobierno abierto" para propiciar su empoderamiento. $\mathrm{Al}$ respecto, es importante destacar que en México se han desarrollado e

* Licenciado en derecho por la Universidad Nacional Autónoma de México; Enlace de Ponencia en el Instituto Nacional de Transparencia, Acceso a la Información y Protección de Datos Personales (INAI), @VAROmXPv.

** Alianza para el Gobierno Abierto, disponible en http://gobabiertomx.org/. 
implementado planes de acción específicos, que contemplan un espacio de diálogo e intercambio de ideas en colaboración con la sociedad civil, aspectos que le han permitido avanzar y cumplir poco a poco con los retos planteados por la AGA, y los cuales se enlistan a continuación:

- Mejora de servicios públicos.

- Aumentar la integridad pública.

- Manejo de recursos públicos con mayor eficacia y eficiencia.

- Construcción de comunicaciones más seguras.

- Aumento de la rendición de cuentas corporativa.

Es grato contar con una colaboración tan completa como lo es la obra Gobierno abierto y el valor social de la información pública, la cual, a lo largo de su contenido, busca llevar de la mano al lector con ideas clave y objetivas que le permitan entender lo que el fenómeno social del gobierno abierto significa, sus objetivos, principios, sus antecedentes, así como aquellos hechos de la realidad social que se encuentran impactando y desarrollando el referido fenómeno. La obra se encuentra integrada por cuatro capítulos presentados de manera lógica para lograr abordar el tema desde un plano general a un plano particular.

El primer capítulo aporta al lector el marco histórico-conceptual a través del cual se exponen los antecedentes y aspectos conceptuales, planes de acción, el marco ideológico del cual parte la existencia y creación de la AGA, así como las razones y objetivos que engloban a dicho fenómeno social. La obra da un enfoque pertinente respecto del papel que juega la información pública para generar un empoderamiento de la sociedad mexicana y con ello desarrollar una de las principales metas del "Gobierno abierto”, que es la participación ciudadana a través del uso de información veraz para el empoderamiento de su sociedad y su efecto en la toma de decisiones.

En el segundo capítulo, la obra enmarca el papel de la participación ciudadana y su efecto en la democracia en México, tomando como base la existencia de la consulta popular y la iniciativa ciudadana, que son herramientas consagradas en el artículo 35 de la Constitución Política de los Estados Unidos Mexicanos. En ese sentido, se presentan hechos históricos que implicaron el ejercicio ciudadano de dichos derechos, resaltando la existencia de antecedentes en la historia de nuestro país, íntimamente relacionados con aquellos elementos que permiten garantizar la participación ciudadana y con ello la posibilidad de llevar a México a cumplir con los objetivos y retos de la AGA. 
Asimismo, derivado del referido capítulo, se proporcionan cifras que reflejan el uso de las tecnologías de la información dentro de las actividades gubernamentales que desempeñan las entidades federativas, resaltando con ello el papel que juega el uso del Internet, las aplicaciones y otras herramientas electrónicas en el desarrollo de lo que denominan como "democracia interactiva" a través del derecho al libre acceso a la información plural y oportuna, y el acercamiento de la sociedad con el gobierno.

En ese sentido, la obra arroja enriquecedores contenidos que permiten evidenciar el desarrollo conjunto a lo largo del territorio mexicano, para implementar aquellas herramientas que colaboran en la implementación del "Gobierno abierto", como lo son el uso de las tecnologías de la información; de ahí que con su uso se vea garantizado el derecho de acceso a la información con que cuenta toda persona.

$\mathrm{Al}$ respecto, se incluye el estudio de caso que ampara la existencia del sistema electrónico Infomex, herramienta utilizada por el Instituto Nacional de Transparencia y Acceso a la Información y Protección de Datos Personales a efecto de atender las solicitudes de acceso a la información y protección de datos personales de los particulares. Este modelo permite el acercamiento entre gobernado y gobierno a efecto de garantizar el ejercicio de su derecho a recibir información de manera facilitada y acceder a los servicios que brinda la autoridad.

Cabe destacar que la obra en comento busca desarrollar dentro de sus capítulos tercero y cuarto el impacto que recientemente ha generado la adopción de las metas expuestas por la AGA a través de los planes de acción desarrollados por el Estado mexicano.

En ese sentido, los colaboradores de la obra aportan un claro ejemplo, a través de tres proyectos implementados a partir de 2011, de las acciones que han obtenido resultados satisfactorios y que al tratarse de hechos sociales de impacto nacional generan certidumbre respecto del camino que ha tomado nuestro país para cumplir con sus compromisos; al respecto, se desarrolla el caso de los "Mecanismos de participación social en guarderías del Instituto Mexicano del Seguro Social”, la implementación del programa social denominado Mejora tu Escuela, y el desarrollo del Programa para Combatir la Mortalidad Materna.

Con lo anterior, se aportan elementos que garantizan la idea de una sociedad empoderada a través de su acercamiento a información útil y veraz ,que le permita atender sus necesidades de una forma sencilla, rápida, y a través del uso de las tecnologías de la información con objeto de combatir y evitar problemas de impacto social. 
Por último, en el capítulo cuarto se proporciona un panorama amplio respecto de los avances que a la fecha se han generado; de manera específica se citan los ejemplos de los estados de Veracruz y Oaxaca, así como el importante papel del Instituto Nacional de Transparencia, Acceso a la Información y Protección de Datos Personales con la implementación del Sistema Subnacional de Gobierno Abierto.

$\mathrm{Al}$ respecto, es de resaltar los esfuerzos conjuntos en la obra, ya que con ello se contribuye para generar una fuente certera que permite aportar conocimiento básico respecto del "Gobierno abierto". Proporciona un panorama amplio respecto de dicho fenómeno aplicado en el caso - mexicano, así como las metas cumplidas y los objetivos que aún faltan

- por atender. En el texto no se pierde de vista que aun cuando el Estado mexicano tiene pocos años como miembro de la AGA, avanza a pasos agigantados en el desarrollo de un sistema gubernamental que empodere a su sociedad a través del acceso a la información pública, con el firme propósito de incentivar el ejercicio de la participación ciudadana y con ello incluir a la sociedad en la toma de decisiones del Estado.

\section{Bibliografía DEL LIBRO RESEÑAdO}

Luna Pla, Issa y Bojórquez Pereznieto, José Antonio (coords.); Hofmann, Andrés (ed.), 2015, Gobierno abierto y el valor social de la información pública, México, Instituto Tabasqueño de Transparencia y Acceso a la Información Pública-UNAM, Instituto de Investigaciones Jurídicas, disponible en http://gobiernoabiertomx.com/. 